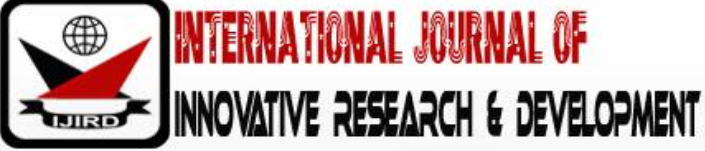

ISSN 2278 - 0211 (Online)

\section{The Mediation Effect of the Semantic Layer on the Relationship between Technical and Information Infrastructure with e- health Interoperability in Ugandan Public Hospitals}

\begin{tabular}{|c|}
\hline Lawrence Nkamwesiga \\
Lecturer, Muni University, Uganda \\
Kituyi Mayoka \\
Assistant Professor, Makerere University Business School (MUBS), Uganda \\
Musa B. Moya \\
Assistant Professor, Makerere University Business School (MUBS), Uganda \\
Ndi Isoh Alain \\
Lecturer, ICT University, Cameroon \\
Clive Tsuma \\
Lecturer, ICT University, Cameroon \\
Victor Mbarika \\
President, ICT University, Cameroon \\
\hline
\end{tabular}

Abstract:

eHealth Interoperability appears to be in its infancy in developing countries including Uganda. The study investigated the Semantic Layer as the mediator on the relationship between Technical and Information Infrastructure with eHealth interoperability in Ugandan Public Hospitals using a quantitative approach. Stratified and simple random sampling were used to select respondents. The study used descriptive statistics and structural equation modelling approach for data analysis. The findings further revealed that all hypotheses, $\mathrm{H}_{1}, \mathrm{H}_{2}$, and $\mathrm{H}_{3}$, significantly explained eHealth Interoperability in Ugandan Public Hospitals. Thus, all the objectives of the study (Objective 1 and 2) were achieved. It is recommended that stakeholders implementing eHealth Interoperability should consider Technical and Information Infrastructure, and Semantic Layer as key factors explaining eHealth Interoperability in Ugandan Public Hospitals. The study further recommends that software developers should adopt Semantic Layer to improve information exchange in Ugandan Public Hospitals. Further, the Ugandan government should adopt key factors of eHealth Interoperability proposed in this study to achieve sustainable development goals and Uganda Vision 2040 goals.

Keywords: Semantic layer, technical infrastructure, information infrastructure, ehealth interoperability, structural equation modelling, Ugandan public hospitals

\section{Introduction}

Health data is generated in various formats that exist in disparity. This concurs with (Lesuer, 2016) that healthcare data exists in different data formats: text, numeric, paper, digital, pictures, videos, and multimedia. According to empirical evidence, the challenges of healthcare data are understanding clinical notes for the inference that at times consist of complex disparate patient sources and handling images that come in big volumes of medical data (Sun \& Reddy, 2013).

The use eHealth interoperability increases the capability of organizations, eHealth solutions, systems or entities to cooperate in the bid to serve the patients' and other health stakeholders' interests. This is achieved through the Semantic Layer that provides the mediation effect for uniform information exchange (Refined eHealth European Framework([REEF], 2015). Thus, eHealth interoperability promotes flexibility, cost-effectiveness, makes virtually seamless systems via the Semantic Layer.

The researchers were motivated to investigate the mediation effect of the Semantic Layer on the relationship between Technical and Information Infrastructure with eHealth Interoperability in Ugandan Public Hospitals. Furthermore, to contribute to theory in the world of academia by introducing the mediation effect of the Semantic Layer.

\subsection{Background}

Historically, the idea of transforming medical records from manual to electronic medical records started on its way back in the mid-1960's (Reid, et al., 2005). A series of transformations in the healthcare industry in the U.S. took place 
until 2004, when the president by then created the Office of the National Coordination for Health Information and Technology, announcing the idea that Health Information Technology should be nationally coordinated (Gur-Arie, 2005). Similar studies were conducted in Europe that led to the development of a European eHealth Interoperability Framework in a bid to support eHealth interoperability across a range of European countries that incorporated policies, standards, recommendations, concepts, principles, and specifications (Langenhove, et al., 2013). There has been a struggle to make eHealth systems interoperable in all Ugandan Public Hospitals (Sandin, 2013).

Contextually, although various initiatives have come up in Uganda, none of them have done enough to explain eHealth Interoperability (Sandin, 2013). Health Data is still generated by manual medical health management information systems that are paper-based and report aggregated data that is not interoperable with the District Health Information Systems-Version 2 (Alunguru, et al.,2015).

eHealth Interoperability is conceptualized in three levels: foundational, structural, and semantic. Foundational level deals Technical Infrastructure while Structural level deals with Information Infrastructure (Health Information and Management Systems Society [HIMSS], 2013; European Commission [EC], 2012). The Sematic level is conceptualized as the level where two or more systems exchange information for meaningful use (EC,2012). Thus, the semantic level improves wellness as well as the quality, safety, cost-effectiveness, and access to healthcare delivery (Sciore, et al., 1994). In this study, the researchers concentrated on the mediation effect of the Semantic Layer on the relationship between Technical Infrastructure and Information Infrastructure with eHealth Interoperability.

Theoretically, two theories guided this study: Semiotic Triangle Theory (Ogden \& Richards, 1923) and Theory of Semantic Values (Sciore, et al., 1994). According to the Semiotic Triangle Theory, a computer system has a point to refer to in a real-world referent as conceived by the people who developed it. Using this concept, the researchers derived two constructs of Technical and Information Infrastructure to explain computer systems and networks in Ugandan Public Hospitals. The researcher used the Theory of Semantic Values to derive two constructs of Semantic Layer and eHealth Interoperability.

\subsection{Problem Statement}

In Ugandan Public Hospitals, Health systems have continued to face challenges on multiple fronts including interoperability and inconsistency (Kitoogo, 2017). Thus, there are inappropriate ways of exchanging patient's data (Alunguru, et al.,2015). Ugandan general hospitals are characterized by duplication of records and efforts as the same patient data is repeated and sometimes lost at every stage of a patient's visit and checkup by different health personnel.

Notwithstanding, the current health management system is dominated by manual recording and storage of patients' records on paper (Alunguru, et al.,2015) leading to high operational costs of health facilities that directly inflate the national health budget. Henceforth, the health management systems in Ugandan public hospitals are disjointed and lack conversion rule and standards making health information exchange inflexible. A situation that has caused additional costs and efforts in trying to share disparate data. Moreover, there seems to be little academic Literature to address eHealth interoperability in Ugandan public hospitals. The researchers sought to investigate how eHealth Interoperability in Ugandan Public Hospitals would be improved while considering the Semantic Layer, Technical and Information Infrastructure as the main determining constructs to come up with an eHealth Interoperability Model.

\subsection{Main Objective}

The general objective was to investigate, applying a quantitative approach, the mediating effect of Semantic Layer on the relationship between Technical Infrastructure and Information Infrastructure with eHealth interoperability in Ugandan public hospitals.

\subsection{Specific Objectives}

- To examine the mediating effect of a Semantic Layer on the relationship between Technical Infrastructure and Information infrastructure with eHealth interoperability in Ugandan public hospitals.

- To assess the relationship between the Semantic Layer and eHealth Interoperability in Ugandan Public Hospitals.

\subsection{Research Questions}

- What is the mediation effect of the Semantic Layer on the relationship between Technical Infrastructure, Information Infrastructure and eHealth Interoperability in Ugandan public hospitals?

- What is the relationship between Semantic Layer and eHealth interoperability in Ugandan public hospitals?

\subsection{Justification}

To the Ugandan government, the study will help in the formulation of polices that are centred around information exchange via a common interface known as the Semantic Layer to achieve SDGs and meet the Ugandan Vision 2040(National Plain Authority [NPA], 2017). Secondly, software may use the concept of Semantic Layer to develop a common interface that may improve information exchange in Ugandan Public Hospitals. Thirdly, Semiotic Triangle Theory (Ogden \& Richard, 1923) and Theory of Semantic Values (Sciore, et al., 1994) both address technological issues and nonexplained clearly the use of Semantic Layer to explain eHealth Interoperability in Ugandan Public Hospitals. Thus, the study added knowledge to the field of academia. 


\section{Review of Related Literature}

The literature review considered journal articles, government reports and policies that had information about eHealth, interoperability levels, data and information exchange theories. The search key words used included information exchange, interoperability, data formats, data structures, mediation effect, Ugandan Public Hospitals. Boolean search words AND and OR were to improve on the search efficiency for articles. The researchers used google scholar and google as the main search engines for journal articles, government reports and polices. The researchers followed the inclusion and exclusion techniques for selecting valid articles. The most recent articles that were quantitative in nature were considered viable. The theories that were of past years were included because they were no recent theories or frameworks for eHealth interoperability. On the other hand, articles that were of the past years were excluded from the search except for those that had information that has not been updated and was considered useful for this study.

\section{1. eHealth Interoperability}

The concept of eHealth interoperability is defined as a platform that meaningfully integrates data or information stored in disparate formats using various proprietary medical information systems, to work as a unit while consuming exchanged information to contribute to more effective and efficient patient healthcare (EC, 2008). It is further indicated that the automatic transfer of information between two or more care sites speeds up service delivery and reduces duplicate testing and prescribing (EC, 2008).

Throughout this study, interoperability in the context of e-health is referred to as e-health interoperability. This is described as a technology whereby health information systems are able to exchange information with each other, regardless of vendor platform and data structure, and also consume that information and make it useful to the medical providers including implementing partners according to international standards and policies.

\subsection{Underpinning Theories}

Theories provide a model for a researcher to think of why the world is the way it is (Strauss, 1995). Theories simplify the world by explaining how it works (Maxwell, 2016). A theory could also be considered as a proven set of propositions commonly regarded as correct. Thus, a theory is considered as a guiding rope that provides more insight into a researcher's study which is in line with literature that theory informs methodology and enables the researcher to link to the research paradigms and philosophies (Crotty, 2003). A conceptual model is a set of concepts, theories, and paradigms that are actually constructed by the researcher according to how the researcher thinks about the phenomenon is and why it is like that.

The Semantic Triangle Theory explains the meaning of symbols as applied in the real world using a three-point triangle notation: symbols, conceptualization, and referent (Ogden \& Richards, 1923). Furthermore, Semiotic Triangle Theory helped the researchers to gain more understanding of information and technical infrastructure in the context of Ugandan genera hospitals. Thus, the theory enabled the researchers to gain more knowledge of how data is captured, stored, and exchanged between disparate systems. The construct of Technical Infrastructure was measured using computer systems and Networks while the construct of information infrastructure was measured using data organization and structure as variables

The researchers adopted the Theory of Semantic Values which states that for systems to exchange two or more values, both must understand the values being exchanged (Sciore, et al., 1994). Semantic Layer and eHealth Interoperability were derived from this theory. The Semantic Layer was used to explain the mediation effect of the Semantic Layer on the relation between Technical Infrastructure and Information Infrastructure with eHealth Interoperability. In this study, the construct of Semantic Layer was measured using the variables of conversion rules and standards while eHealth Interoperability was measured using effectiveness of eHealth Interoperability.

\subsection{Theoretical Gap Analysis}

The two theories explained above were triangulated and analysed to identify the theoretical gap by using a matrix analysis technique. The Semiotic triangle theory is concerned with the organization of symbols using standard rules known as syntax in the context of Information Infrastructure and the computer systems that are used to send data over a given network (Ogden \& Richards, 1923). However, the Semiotic Triangle Theory does not address Semantic Layer and eHealth Interoperability. Therefore, the symbols contained in the computer systems may be exchanged between systems, regardless of meaning, and hence, the need for the Semantic Layer.

The Theory of Semantic Values argues that data exchange between disparate systems of the organization should take place using standards and protocols over a system architecture that will convert data into meaningful use for consumption by both systems and users (Sciore, et al., 1994) This concurs with empirical literature that systems that are not interoperable always conflict and can be disastrous (Siegel, et al., 1991). While this appears to be true, (Sciore, et al., 1994; Siegel, et al., 1991), Theory of Semantic Values (Sciore, et al., 1994) does not address Technical and Information Infrastructure. The theoretical gap analysis is summarized Table 1. 


\begin{tabular}{|c|c|c|}
\hline & $\begin{array}{c}\text { Semiotic Triangle Theory } \\
\text { (Ogden \& Richards, 1923). }\end{array}$ & $\begin{array}{c}\text { Theory of Semantic Values } \\
\text { (Sciore, et al., 1994) }\end{array}$ \\
\hline Technical Infrastructure & $\sqrt{ }$ & $\mathrm{X}$ \\
\hline Information infrastructure & $\sqrt{ }$ & $\sqrt{ }$ \\
\hline Semantic layer & $\mathrm{X}$ & $\sqrt{ }$ \\
\hline eHealth interoperability & $\mathrm{X}$ & \\
\hline
\end{tabular}

Table 1.Gap analysis Matric Analysis

$\mathrm{X}=$ No, $\quad \sqrt{ }=$ Yes

\subsection{Conceptual Model}

The proposed conceptual model is a combination of constructs from two theories: Semiotic Triangle Theory (Ogden \& Richards, 1923) and the Theory of Semantic Values (Sciore, et al., 1994).

We applied the Semiotic Triangle Theory (Sciore, et al., 1994) to assume that eHealth devices and systems interpret electronic health records that has been structured to a certain format syntactically. This explained the construct of Information Infrastructure with two variables of data organization and structure. Furthermore, this theory explained the construct of Technical Infrastructure that gave two variables of computer system and network.

Furthermore, it is argued that eHealth systems should be able to attach meaning to the received information, interpret it, and use it meaningfully for semantic operation (Sciore, et al., 1994). In order to achieve this, the systems themselves need to have interfaces installed in them that enable seamless exchange information without a human interface that would otherwise cause additional costs (European Telecommunications Standards Institute [ETSI], 2008).

The researchers posited that eHealth interoperability would be realized where devices, systems, or applications are able to exchange information; data is structurally organized; and information is exchanged meaningfully. Thus, leading to the conceptual model shown in Figure 1.

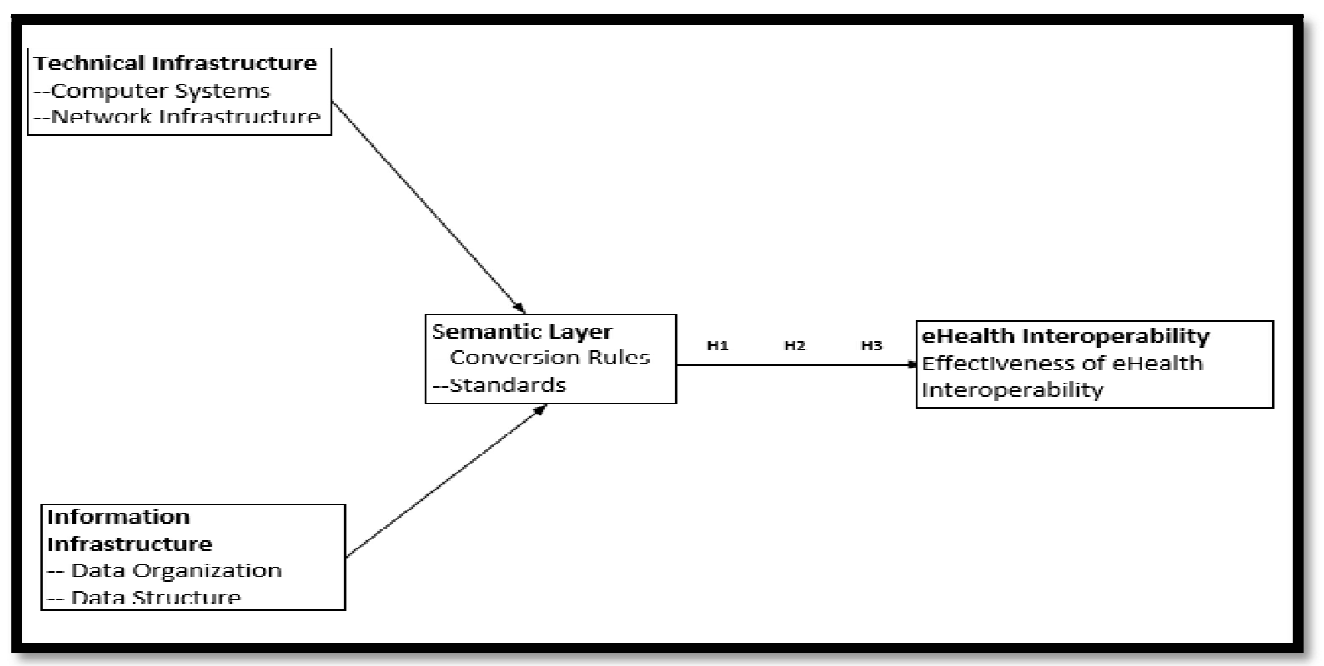

Figure 1: Conceptual Model

\subsection{Research hypotheses}

From the above discourse, the following hypotheses were developed.

- H1: Semantic Layer mediates the relationship between Technical Infrastructure and eHealth Interoperability in Ugandan Public Hospitals.

- H2: Semantic Layer mediates the relationship between Information Infrastructure and eHealth Interoperability in Ugandan public hospitals

- H3: There is a significant positive relationship between Semantic Layer and eHealth Interoperability in Ugandan Public Hospitals.

\section{Research Methodology}

\subsection{Research Philosophy, Design, and Perspectives}

The study adopted the philosophy of positivism guided by deductive reasoning approach, quantitative design, and descriptive survey strategy. Positivism philosophy was used because it focuses on universalism and single reality (Creswell, 2012). Thus, we applied positivism to assume that all respondents are the same and collected parametric data independently from the public hospital health workers and patients. Notwithstanding, the researchers used Ontological 
perspective to select research sites and respondents while Epistemological perspective was used collect data, that was used to generate information, from the respondents (Creswell, 2012). On the other hand, Axiological perspective was used in research ethics consideration. The researcher used a cross-sectional study where data were collected at one time as explained in the Research Onion Theory (Saunders, et al., 2009). The cross-section study was used because the longitudinal study lacks control (Creswell, 2012).

\subsection{Research Site, Description of the Population, and Sample}

We selected three general hospitals of Kitagata, Naguru, and Nebbi. The distribution of the study cases was based on location whereby the researchers wanted to observe eHealth practices in regard to eHealth Interoperability, in the city, town, and rural public hospitals. The study population of approved health workers was 453 . The research participants for this study included health workers and patient that gave a total of 270 participants.

The known sample was obtained from standard statistical tables that have been used by many researchers and have proved to give consistent results over time (Delice, 2010; Cochran, 1923). From the tables, the known population sample size was 42 administrative health workers and 132 health workers from the three hospitals selected. The unknown population of 96 patients was computed using a standard proportionality formula given below (Cochran, 1923).

Sample Size $=\frac{\mathrm{z}^{2} * \mathrm{pq}}{\mathrm{e}^{2}}$;

\subsection{Sampling Strategies}

We used probabilistic sampling, which assumes that each unit in a population has a specifiable chance of being selected. The researchers employed multi-stage sampling approach including stratified sampling and simple random sampling techniques, which is classified under probabilistic sampling. Stratified sampling was used to divide the study population into groups of medical staff and patients in Ugandan Public Hospitals. This was preferred because the aforementioned two groups are heterogeneous and it minimizes errors (Goel, 2014). We also used simple random sampling to select participants from each group, i.e. medical staff and patients. Simple random sampling has the advantage that participants have equal chances of being selected within a given sample (Ajay, 2018).

\subsection{Data Sources}

Primary data were collected from respondents including hospital health workers and patients. This concurs with (Hox, et al., 2005) that primary data are data that comes from the specific phenomenon under investigation and are provided by the participants. On the other hand, secondary data sources included information from peer-reviewed articles published in journals; government publications and reports, and reports published by departments of health, health implementing partners' information published by the ministry of education, Health research experts and consultants.

\subsection{Data Management and Analysis}

The researchers checked the data for consistency. After checking, the researchers entered the data into the statistical package for social scientists (SPSS version 23) computer program for analysis. Respondents have a tendency of skipping some items, a situation that causes some values to miss. Missing values need to be identified and managed as they affect multivariate analysis (Hair, et al., 1998). We adopted Little's test to check for missing values. The commonly used method of test for cases Missing Completely at Random mechanism which assumes that missingness is not related to any variable in the dataset or variable of interest (Garison, 2015). Finally, we analysed data using Structural Equation Modelling and descriptive statistics using Analysis of Moment of Structures version 23 and SPSS version 23.

\section{Findings}

\subsection{Respondents' gender, Age Group, and Category}

The results indicated that most of the respondents were female (frequency=157, $65.1 \%$ ). Male respondents were approximately 34.9 percent. The results could be due to the fact that most patients who came to the hospital were female and that there are more female medical workers than males.

Furthermore, indicated that most of the respondents in the quantitative study were in the age group of 18-27 (Freq=116, 48.1\%), followed by those in age group 28-37 (Freq=77, 32.0 \%) and age group 38-47 (Freq=36, 14.9). Only 12 respondents, constituting $5 \%$ were 48 years of age and above.

Most of the respondents in the study were medical workers (Freq $=122,50.6 \%$ ), followed by patients (Freq=89, $36.9 \%)$. The administrators constituted a frequency of 30 (12.4\%). The expected number of respondents was 270. However, only 241 participants responded, giving a response rate of 89.3 percent.

\subsection{Structural Equation Model Results: Mediation Effect}

The mediating variable stands in between an independent and dependent variable. The mediation model explains the influence of causal variable, also known as an independent variable, on a mediating variable and in turn the influence of the mediator variable on the dependent variable (Hayes, 2013). 


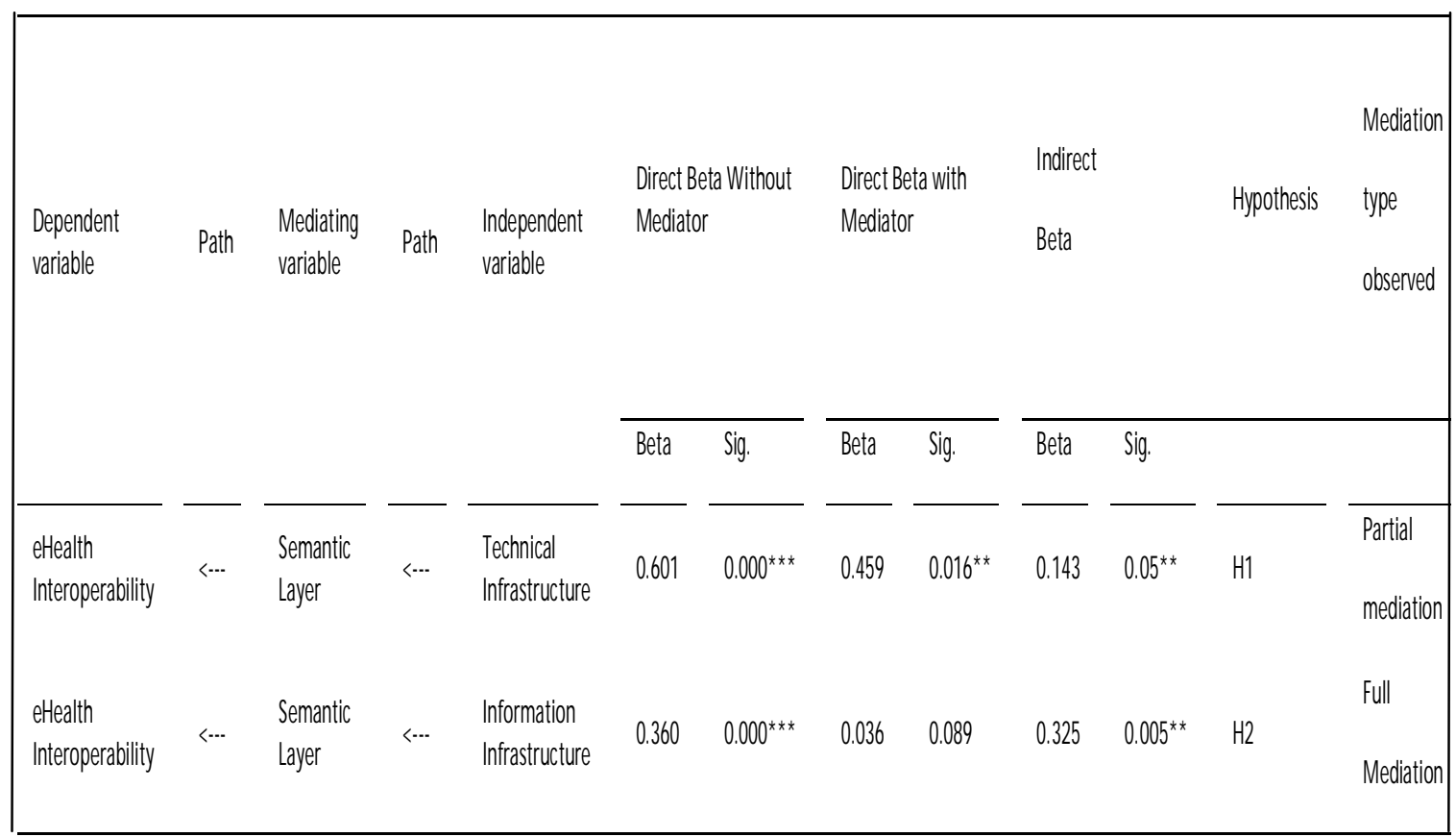

Table 2: Mediation Results

**Significant $\mathrm{P}<=0.05,{ }^{* * *}$ Significant $\mathrm{P}<=0.001$

We also adopted conditions for mediation in line with (Hayes, 2013).

\subsubsection{Condition 1-No Mediation}

If indirect effect is not significant. The results from Table 2 indicate that the indirect Beta for Information infrastructure is significant (Beta $=0.325, p=0.005$ ) while for Technical Infrastructure (Beta $=0.143, p=0.05$ ), thus the mediation exists.

\subsubsection{Condition 2-Partial Mediation}

If direct (with mediator) and indirect are significant. Results from Table 2 above indicate that direct Beta with mediator for Information infrastructure is insignificant (Beta $=0.036, p=0.089$ ) and indirect Beta is significant (Beta $=0.325$, 0.005), indicating no partial mediation. For Technical Infrastructure, direct Beta with mediator is significant (Beta $=0.459$, $\mathrm{p}=0.016$ ) and indirect Beta is significant ( $\mathrm{Beta}=0.0143, \mathrm{p}=0.05$ ), indicating partial mediation.

\subsubsection{Condition 3-Full Mediation}

If indirect is significant and direct (with mediator) is not significant. The results from Table 2 indicate that indirect is significant (Beta $=0.325, \mathrm{p}=0.005$ ) and direct (with mediator) is not significant (Beta $=0.036, \mathrm{p}=0.089$ ), indicating full mediation. Thus, the Semantic Layer has both partial and full mediation effect on the relationship between Information Infrastructure and eHealth Interoperability (Hayes, 2013).

\subsubsection{Condition 4}

If direct effect of independent variables (Information and Technical Infrastructure) on Semantic Layer(mediator) is significant or if direct effect from Semantic Layer to eHealth Interoperability (dependent variable) is significant. Accordingly, and in line with condition 4, the researchers employed SEM, in particular SPSS AMOS version 23, as a confirmatory test for significance of the mediation effect.

\subsection{Structural Equation Model Results for the Measured Model}

\begin{tabular}{|c|c|c|c|c|c|c|c|}
\hline \multicolumn{3}{|c|}{ Path } & B & S.E. & C.R. & Beta & P \\
\hline Semantic Layer & $<---$ & Technical Infrastructure & 0.381 & 0.097 & 3.922 & 0.246 & $* * *$ \\
\hline $\begin{array}{c}\text { eHealth } \\
\text { Interoperability }\end{array}$ & $<---$ & Semantic Layer & 0.687 & 0.021 & 33.045 & 0.595 & $* * *$ \\
\hline Semantic Layer & $<---$ & Information Infrastructure & 0.771 & 0.086 & 8.948 & 0.562 & $* * *$ \\
\hline
\end{tabular}


The results in Table 3 confirms that the Semantic Layer mediates the relationship between Information and Technical Infrastructure eHealth Interoperability. Thus, there is a significant positive relationship between Technical Infrastructure and Semantic Layer in Ugandan Public Hospitals (Beta $=0.246^{* * *}$ ). Similarly, the results indicate that there is a significant positive relationship between Information Infrastructure and Semantic Layer in Ugandan Public Hospitals (Beta $=0.562^{* * *}$ ). Also, the findings indicate that there is a positive significant relationship between Semantic Layer and eHealth Interoperability in Ugandan Public Hospitals (Beta $=0.595^{* * *}$ ). Therefore, condition 4 is fulfilled. The supported model is as indicated in Figure 2.

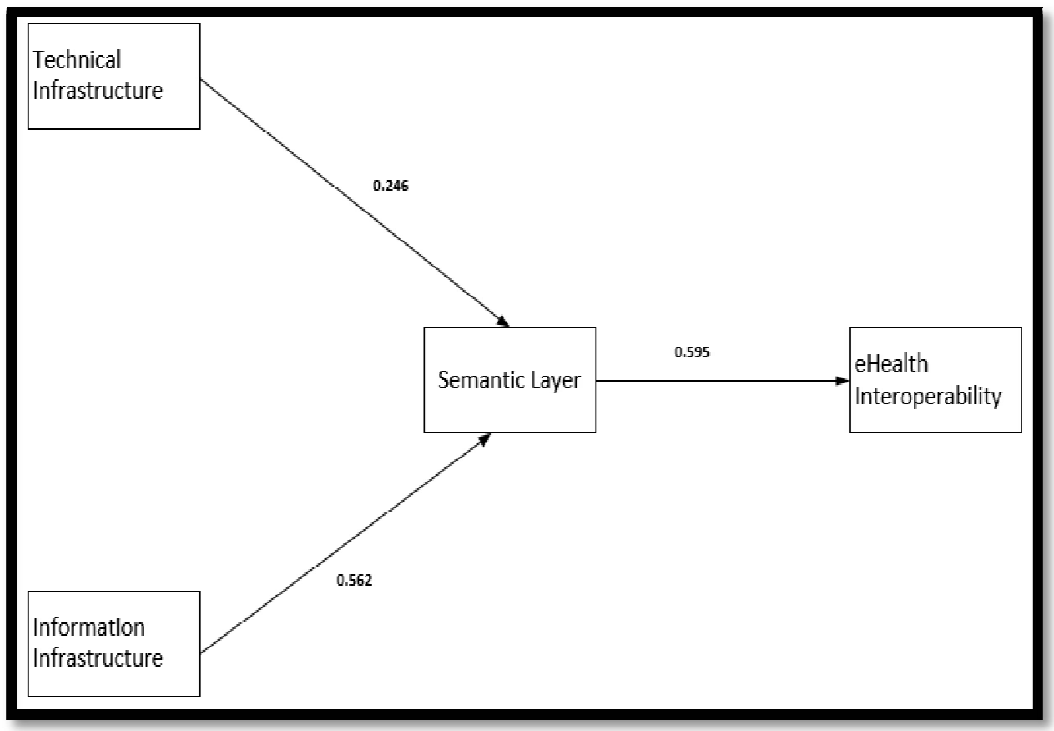

Figure 2: Measured Model

- $\mathrm{H}_{1:}$ Semantic Layer mediates the relationship between Technical Infrastructure and eHealth Interoperability in Ugandan Public Hospitals

The results in Table 2 indicate that the indirect effect of Semantic Layer on the relationship between Technical Infrastructure and eHealth Interoperability are significant (Beta $=0.143, p=0.05$ ), while the direct effects of the model with the mediator are also statistically significant, (Beta $=0.459, p=0.016$ ), a condition for partial mediation (Hayes, 2013). $H_{1}$, was accepted since it was supported by data.

- $\mathrm{H}_{2}$ : Semantic Layer mediates the relationship between Information Infrastructure and eHealth Interoperability in Ugandan Public Hospitals

Results in Table 2 indicate that the indirect effects of Semantic Layer on Technical Infrastructure and eHealth Interoperability are statistically significant (Beta $=0.325, p=0.005$ ), while the direct effects of the model with the mediator are statistically not significant (Beta $=0.036, p=0.089$ ), a condition for full mediation (Hayes, 2013). Therefore, $\mathrm{H}_{1}$ was accepted, since it was supported by data.

- $\mathrm{H}_{3}$ : There is a significant positive relationship between the Semantic Layer and eHealth Interoperability in Ugandan Public Hospitals

There was a significant positive relationship between Semantic Layer and eHealth Interoperability in Ugandan public hospitals (Beta $=0.595, \mathrm{p}<0.001$ ) as shown in Table 3 above. This means that for a unit change in the Semantic Layer, there is a corresponding increase of 59.5 percent in the eHealth Interoperability in Ugandan Public Hospitals. Thus, $\mathrm{H}_{1 \mathrm{c}}$ was accepted since it was supported by data.

\section{Discussion of Findings, Conclusion, and Recommendations}

- $\mathrm{H}_{1}$ : Semantic Layer mediates the relationship between Technical Infrastructure and eHealth Interoperability The findings indicate that the data exchanged by health workers and patients in Ugandan Public Hospitals can partially be controlled by Semantic Layer for interpretation. This is in line with empirical literature that the Semantic Layer has a set of protocols that assist in the interpretation of the exchanged messages for meaningful use (Hayes, 2013). Thus, Technical Infrastructure has a significant partial effect on eHealth Interoperability, meditated by the Semantic Layer. This means that increasing Technical Infrastructure in Ugandan Public Hospitals will significantly and partially increase eHealth Interoperability via the Semantic Layer.

- $\mathrm{H}_{2}$ : Semantic Layer mediates the relationship between Information Infrastructure and eHealth Interoperability The findings further indicate that Information Infrastructure has indirect a significant causal indirect effect on eHealth Interoperability meditated by the Semantic Layer. In other words, stakeholders including health workers and patients understand that data or information exchanged under appropriate standards and rules will improve interoperability in Ugandan Public Hospitals. This agrees with the empirical literature that for interoperability to be achieved, information should be exchanged meaningfully (LeSuer,2016). From the findings, the Semantic Layer fully mediates the relationships between Information Infrastructure and eHealth Interoperability in Ugandan Public Hospitals. Thus, the Semantic Layer will resolve the issue of disparity to improve information exchange for meaningful use. 
- $\mathrm{H}_{3}$ : There is a significant positive relationship between Semantic Layer and eHealth Interoperability in Ugandan public hospitals.

The results indicate that there is a significant positive relationship between Semantic Layer and eHealth Interoperability in Ugandan Public Hospitals. This is in agreement with empirical literature that Semantic Layer consists of a set of protocols and standards that facilitate seamless exchange of information, thus, improving eHealth Interoperability by adding meaning to the data (Van der veer \& Wiles, 2008).

The results indicate that in Ugandan public hospitals, the health workers and patients understand that Semantic Layer will improve the exchange of data and information for meaningful use. Therefore, an improvement in the set of standards or rules will lead to a corresponding improvement in eHealth interoperability that will in turn lead to improved healthcare service delivery in Ugandan public hospitals.

\subsection{Conclusions}

The purpose of this study was to investigate two specific objectives. The main objective was to examine the mediating effect of a Semantic Layer on the relationship between Technical and Information infrastructure with eHealth interoperability in Ugandan public hospitals. From the findings above, we conclude that the Semantic Layer plays a significant mediation role in the relationship between Technical and Information Infrastructure with eHealth Interoperability in Ugandan public hospitals. The findings further revealed that all hypotheses $\left(\mathrm{H}_{1}, \mathrm{H}_{2}, \mathrm{H}_{3}\right)$ significantly explained eHealth Interoperability in Ugandan Public Hospitals. Thus, objectives 1 and 2 were achieved. Therefore, improving Technical Infrastructure will partially improve eHealth Interoperability via the Semantic Layer. Therefore, improving computer systems, Network infrastructure, Data Organization and Structures will have a partial and full causal effect on the effectiveness of eHealth Interoperability in Ugandan Public Hospitals.

\subsection{Implication to Theory}

The final model in this study adapted two theories: Semiotic Triangle Theory and Theory of Semantic Values, to explain the mediation effect of Semantic Layer on the relationship between Information Infrastructure and eHealth Interoperability in Ugandan Public. The Semiotic Triangle Theory (Ogden \& Richard, 1923) and Theory of Semantic Values (Sciore, et al., 1994) both address technological issues. However, these theories do not use Semantic Layer to clearly explain eHealth Interoperability in Ugandan Public Hospitals. Therefore, we can say that the study has a significant theoretical contribution to both the Semiotic Triangle Theory and Theory of Semantic Values when applied to eHealth interoperability in Ugandan Public Hospitals. Thus, the study has contributed significantly to the realization of eHealth Interoperability using a new approach of Semantic Layer, which has never been used, thus, contributing to knowledge development.

\subsection{Implications and recommendations to practice}

As established by this study, Semantic Layer plays a significant role in improving eHealth interoperability in Ugandan Public Hospitals. This is evidenced by the results of this study that Semantic Layer fully mediates the relation between Information Infrastructure and eHealth Interoperability. Meaning that without the Semantic Layer, information may be meaningless to users in Ugandan Public Hospitals. Therefore, from the observations of this study, it is recommended that stakeholders implementing eHealth Interoperability in Ugandan Public Hospitals should improve Technical and Information Infrastructure while putting more emphasis on Semantic Layer. Once still, software developers need to address issues of interoperability by embedding Semantic Layer that would work as an application programming interface in all computer systems and Network Infrastructure in Ugandan Public Hospitals. This will help in the interpretation of data regardless of the application producing it.

\subsection{Implications and recommendations to Policy}

The Ugandan government should formulate policies focused on eHealth interoperability while considering Information Infrastructure and Semantic Layer. This may help the government to achieve its national goals of interoperability for all government systems as recommended in the 2040 vision (NPA, 2017).

\section{Limitations of the Study}

The study used quantitative inquiry, which makes conclusions basing on parametric statistical data using deductive reasoning, as opposed to inductive reasoning addressed by qualitative inquiry. Although it was necessary to use quantitative approach given that the sample was big, the approach uses observations and assumptions that are quantified. Some significant themes that might have been revealed by the subjective inquiry could have been left out by the objective inquiry.

\section{Areas for Future Improvement}

Given the limitations above, future research should be conducted using a qualitative inquiry. For instance, a case study would be conducted with a group of medical workers involved in policy formulation and implementation. Further research should be conducted to examine the factors influencing eHealth Interoperability in Ugandan Public Hospitals other than Information Infrastructure and Semantic Layer. 


\section{References}

i. $\quad$ Ajay, S.S., \& Micah, \& Micah, B. (2018). Sampling Techniques \& Determination of Sample Size in Applied Statistics Research: An Overview. International Journal of Economics, Commerce and Management United Kingdom, vol. (11). Retrieved February 7, 2018, from http:/ / ijecm.co.uk/ wp-content/ uploads/ 2014/ 11/ 21131.pdf

ii. Alunguru, E., Martin, M. Mbonye, M.M.., Tumwesigye, P. Julian Natukunda J., \& Gladys Tugume, G.T(2015). Strengthening Uganda's health management Information systems at selected public Healthcare facilities. Retrieved August 4, 2017, from http:/ / sustainuganda.org/ sites/ sustainuganda.org/ files/ SUSTAINTechBrief_HMIS FINAL.pdf Cochran, W. G. (1963). Sampling Techniques, 2nd Ed., New York: John Wiley and Sons, Inc.

iii. Creswell, W.J. (2012). Planning, Conducting and Evaluating Quantitative and Qualitative Research. Educational Research, (4th ed). Educational Research.

iv. Crotty, M. (2003). Introduction: the research process. The Foundations of Social Research: Meaning and perspective in the research process. https:// salbayrak.yasar.edu.tr/ wp-content/ uploads/ 2012/ 09/ Lecture-1Reading-Crotty-chapter-1.pdf eHealth Network (2015). Refined eHealth European Interoperability Framework. Retrieved October 1, 2017, from

v. https:/ / ec.europa.eu/ health/ / sites/ health/ files/ ehealth/docs/ ev_20151123_co03_en.pdf

vi. European Telecommunications Institute. (2008). European Telecommunications Standards Institute (ETSI), White Paper, vol. (3). Retrieved from

http:/ / www.etsi.org/ images/ files/ ETSIWhitePapers/ IOP\%20whitepaper\%20Edition\%203\%20final.pdf European Commission (2012). eHealth Action Plan 2012-2020 - Innovative e healthcare for the 21st century.

vii. Garson, D.G (2015). Missing Values Analysis \& Data Imputation. North Caroline, State University: School of Public and International Affairs Statistical Association Publishing: Blue Book Series. Retrieved from http:/ / www.statisticalassociates.com/ missingvaluesanalysis_p.pdf

viii. Goel, B. (2014). Stratified Sampling. Regional Training Course on Sampling Methods for Producing Core Data Items for Agricultural and Rural Statistics. Retrieved from http://www.unsiap.or.jp/ elearning/ el_material/Agri/rap_Sampling_Indonesia/ 8_M2_Stratified\%20Sampling.pdf

ix. Gur-Arie, M. (2013). The History of Healthcare Interoperability.

x. http:/ / hitconsultant.net/ 2013/ 04/ 11/ history-of-healthcare-interoperability/

xi. Hair, J. F. Jr., Anderson, R. E., Tatham, R. L. \& Black, W. C. (1998). Multivariate Data Analysis (5th ed.), New Jersey, Prentice-Hall.

xii. Health Information and Management Systems Society (2013). Definition of Interoperability.

xiii. Hox, J.J. \& Boeije, R.H. (2005). Data collection: Primary vs. Secondary. Retrieved October 30, 2017, from http:/ / www.joophox.net/ publist/ ESM DCOL05.pdf

xiv. Kitoogo, E. F. (2017). Overview of ICT Policies and Strategies in Other Sectors and a Case Study of Health Ministry of ICT and National Guidance.

xv. $\quad$ Langenhove, P.V., Decreus, K., Rogala, A., Olyslaegers, T., \& Whitehouse, D. (2013). eHealth European Interoperability Framework European Commission-ISA Work Programme. European Union. Retrieved from

xvi. https:/ / www.uems.eu/ _data/ assets/pdf_file/ 0010/ 1504/ eHealth_European_Interoperability_Framework_Study_report_-July_2013.pdf

xvii. $\bar{L}$ LeSueur, D. (2016). $\overline{5}$ Reasons Healthcare Data Is Unique and Difficult to Measure. HealthCatalyst. Retrieved from https:/ / www.healthcatalyst.com/ 5-reasons-healthcare-data-is-difficult-to-measure

xviii. Maxwell, A.J. (2016). Designing a Qualitative Study. Qualitative Design: An Iterative Approach. Sage Publication.

xix. National Planning Authority (2017). Ugandan Vision 2040. Retrieved November 17, 2017, from http:/ / npa.ug/ uganda-vision-2040/

xx. Ogden, C. K., \& Richards, I. A. (1923). The Meaning of Meaning. 8th Ed. New York, Harcourt, Brace \& World, Inc.

xxi. Reid, P.P., Compton, W. D., Grossman, H.J., \& Fanjiang, G. (2005). Building a Better Delivery System. A New Engineering/Health Care Partnership. Washington, DC: The National Academies Press. https:/ / doi.org/ 10.17226/ 11378

xxii. Sandin, C. (2013). Uganda Health Sector and Partnership Opportunities. Retrieved from http:/ / www.Sandin.se/ Portals/ Sandin/ Documents/ Uganda-Health-Sector-and-

xxiii. Siegel, M., Madnick, S., \& Gupta, A. (1991). Composite information systems Resolving semantic heterogeneities. In Workshop on Inform atzon Tech nology Systems

xxiv. Sciore, E., Siegel, M., \& Rosenthal, A. (1994). Using semantic values to facilitate interoperability among heterogeneous information systems. ACM Transactions on Database Systems (TODS) TODS Homepage archive, v.19 (2), p. 254-290. Retrieved June 30, 2017, from http:/ / dl.acm.org/ citation.cfm?id=176570

xxv. Saunders, M., Lewis, P., \& Thornhill, A. (2009). Formulating the research design, pp.138. Research Methods for Business Students, 5th Ed. Pearson Education Limited. Retrieved October 14, 2017, from

xxvi. https:/ / is.vsfs.cz/ el/ 6410/ leto2015/ BA_BSeBM/ um/ um/ Research_Methods_for_Business_Students_5th_Edition. pdf

xxvii. Saunders, M., \& Tosey, P. (2013). The Layers of Research Design. Retrieved October 29, 2017, from https:/ / anlp.org/ files/ research-onion-layers_42_357.pdf

xxviii. Saunders, M., Lewis. P., Thornhill A., \& Bristow, A. (2016). Understanding research philosophy and approaches to theory development, ch.4, pp.122-161. Retrieved October 14, 2017, from 
https:/ / www.academia.edu/ 13016419/ Research_Methods_for_Business_Students_Chapter_4_Understanding_res earch philosophy_and approaches_to_theory_development

xxix. Strauss, A. L. (1995). Notes on the nature and development of general theories. Qualitative Inquiry 1, 7-18.

xxx. Sun, J., \&Reddy, K.C. (2013). Big Data Analytics for Healthcare. Retrieved October 1, 2017, from https:/ / www.siam.org/ meetings/ sdm13/ sun.pdf

xxxi. Van der Veer, H.., \& Wiles, A. (2008). Achieving Technical Interoperability: the ETSI Approach. Retrieved from http:/ / www.etsi.org/ images/ files/ ETSIWhitePapers/ IOP\%20whitepaper\%20Edition\%203\%20final.pdf 